\title{
Naturally Occurring Hairlines in Orientals of Southeast and East Asian Origin and Their Application in Hair Restoration Surgery
}

\author{
Tyng Yuan Tan, MBBS, Damkerng Pathomvanich, MD, FACS Bangkok, Thailand tyngyuan@hotmail.com
}

\section{Introduction}

The most common cause of male pattern baldness (MPB) is androgenetic alopecia (AGA). ${ }^{1}$ The clinical onset of baldness in both men and women generally occurs at around the age of 30 to 40 years old. ${ }^{2}$ In Southeast and East Asia (SE and E Asia), the incidence of male pattern baldness varies depending on region, is generally lower compared to their Caucasian counterpart, but is also increasing with age..$^{3-10}$ Not surprisingly, more Orientals are seeking a permanent solution to their hair loss by means of hair transplantation. The creation of a natural hairline is possibly one of the most important steps of a successful hair transplant and reflects on the surgeon's aesthetic and artistic flare. Knowing the trend of hairline as it changes with age helps the surgeon to plan ahead and design a hairline that is most natural for the patient. To create a good hairline, understanding the anatomical differences between the Caucasian and Asian hairline is essential. East Asian men tend to have brachycephalic or round skulls, while Caucasians tend to have doliocephalic or ovoid skulls (Figure 1). East Asian scalps tend to be wider and flatter, with rounded frontotemporal angles, and most patients of East Asian origin seek a flatter hairline that mimics this nature. ${ }^{11}$

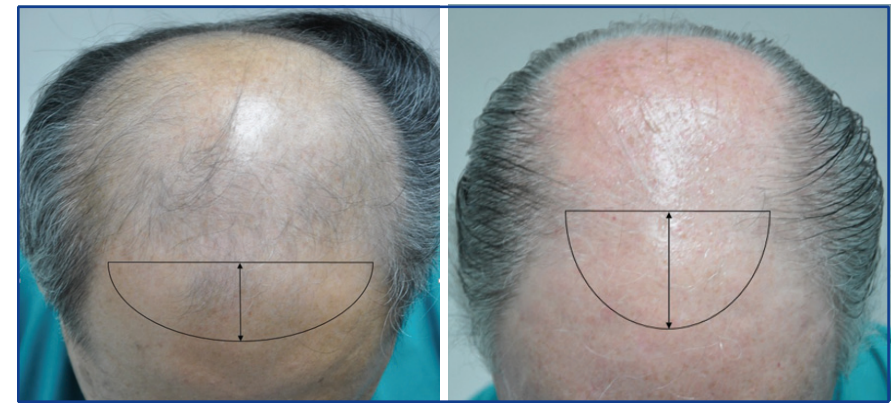

Figure 1. Comparative anatomy of typical Asian and Caucasian skulls (top view). The typical East Asian male has a round skull (left), while the average Caucasian male has an ovoid skull shape (right). (Photos courtesy of DHT Clinic, Thailand)

The angle at which the hair exits the scalp is also important. It is usually at a wider angle in Asians, approximately at a $45^{\circ}$ angle to the skin, but it can be in the range of $45^{\circ}-90^{\circ}$. Caucasians tend to have flatter/more acute hair angles. It is harder to gauge the hair angle in patients with wavy hair. Hair at the temple is much flatter in nature, almost parallel to the skin in Caucasians but not in Asians.

Tsai et al. (2002) reported that the average hair density of the frontal scalp in non-balding Oriental men (ages 20-50) was $84.21 \mathrm{FU} / \mathrm{cm}^{2}$ and that of the temporal scalp was $54.47 \mathrm{FU} / \mathrm{cm}^{2} .^{12}$

\section{Planning the Hairline}

Imagawa (2010) suggested that the ideal hairline should take into consideration the following: ${ }^{13}$

1. Basic facial shape: square, oval, round, triangular, heart shaped

2. Slope of forehead: upslope (common in Caucasian), flat (common in Asians), or downslope (commoner in AfroCarribeans)
3. Frontotemporal angle: Typically lies on a line drawn vertically from the lateral epicanthi of the eye to meet the frontal hairline. Asians tend to have a wide frontotemporal angle compared to the more acute ones seen in Caucasians.

4. Position of hairline: Usually is lower than in Caucasians. In Asians, a mid-frontal point $7-9 \mathrm{~cm}$ from the glabella is usually acceptable, depending on age and projected future hair loss. Strong temples are required to balance a low hairline.

Hair direction refers to the direction in which the hair wants to naturally fall. Beehner (2008) and Unger (2004) described the most common hair direction in Caucasians at the frontal hairline to be from left to right with gradual conversion to anterior (Figure 2). ${ }^{14,15}$ By following the correct hair direction during hair surgery, the surgeon can avoid injuring existing hair and can minimize the risk of shock loss. ${ }^{14}$

Other variations of the hairline commonly encountered in hair restoration surgery include the conjugate "ridgeback," where the hairline on the left and right converge at the center. This is commonly seen in Caucasians but not in Asians (Figure 3). Another variation commonly seen in hair transplantation is a straight hairline on the left and an oblique hairline pointing towards the midline on the right (Figure 4).

Unger also noted the presence of a retained "frontal cowlick" in some patients, which needs to be taken into consideration and followed if the residual hair is strong. ${ }^{15}$ The presence of a widow's peak is common in men and is

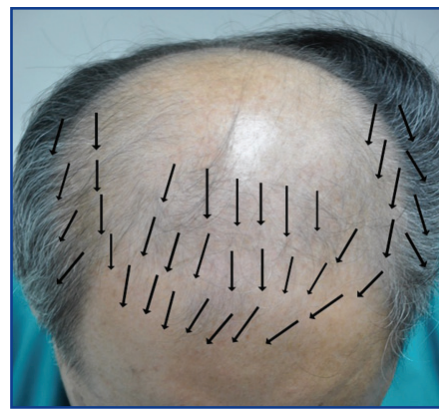
Figure 2. Left-to-right hair direction extending to mid-frontal section, with gradua conversion to straight anterior on right side.

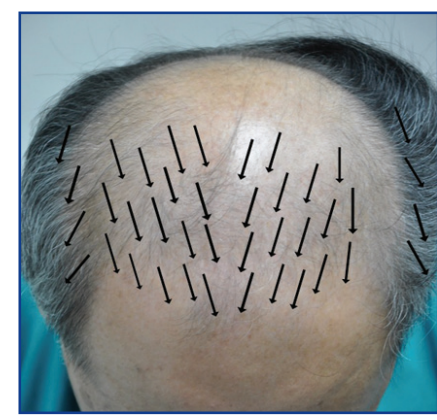

Figure 3. A conjugate "ridgeback" hairline, where there is convergence of the hairs towards the center.

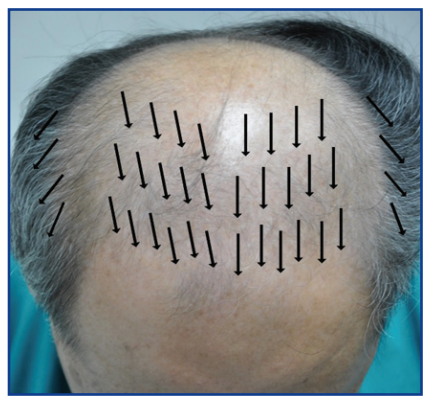

Figure 4. A straight hairline on the left the right. with hair directed towards the midline on frequently an added on feature in hairline design. ${ }^{14}$

There is limited literature on the characteristics of the naturally occurring hairline in Orientals. We studied the changes to the hairline with age in Chinese males and its common characteristics for better application to SE and E Asian patients seeking hair restoration surgery. 


\section{Methodology}

From September to November 2015, we studied a total of 209 Oriental men of SE and E Asia origin (Singapore, Malaysia, Indonesia, China, and Brunei) without AGA (Norwood I-II). Subjects between 18-85 years old were recruited from outside major shopping malls and train stations, bus stations and interchanges, community centers, and Chinatown in Singapore. We looked at the impact on the hairline as one ages, and its associated risk factors (smoking, family history, diet, age, etc.).

Patients age 18 years and above were recruited in the study as androgenetic alopecia becomes clinically apparent after the onset of puberty. ${ }^{10}$ The hairline in the absence of AGA in the majority of the population in the specific age group is the desired hairline that we would want to re-create in hair transplant patients.

Hairline characteristics were examined, including the direction of the hairline; the density and hair count at the first 1.5 centimeters of the frontal hairline and peaks. The mid-frontal point (distance between the interception of a horizontal line between the eyebrows, and a vertical line drawn from the glabella to the anterior-most part of the hairline) and frontotemporal point (distance between the lateral canthus to the anterior-most point of the temporal hairline) were also calculated.

The presence of a widow's peak or cowlick was noted, as well as the natural direction of the hairs at the hairline, and the frontotemporal angle (round/straight), the hair direction at the temples, and the width of the sideburns and its relationship to the tragus were investigated.

Shapiro $(2004,2013)$ described macro irregularity of the hairline that can be seen from a distance that consists of three "mounds" that included one central (consisting of the widow's peak) and two lateral "mounds." The presence of lateral mounds in Asians was also investigated in this study. ${ }^{16,17}$

\section{Results}

We decided to stop data collection at approximately 30 patients per age bracket as the findings were consistent and the recruitment of larger numbers in the study would be an arduous process and would provide little additional input.

\section{Risk Factors and Hair loss}

Of the 209 subjects, $85.7 \%$ have no family history of hair loss, emphasizing the significance of genetics in androgenetic alopecia. There were $49.75 \%$ classified as Norwood I and $50.25 \%$ as Norwood II. Of the participants, $46.9 \%$ were smokers and a majority $(84.7 \%)$ of them were on a mixture of eastern and western diet.

\section{Density}

Hair density decreases as men age (see table below for hairline density of non-androgenetic alopecia men between the ages of 18-85 [n = number of subjects]). The average frontal hairline density in men is $77.8 \mathrm{FU} / \mathrm{cm}^{2}$ and 110 hairs $/ \mathrm{cm}^{2}$. This translates to 1.4 hairs/FU in the first $1.5 \mathrm{~cm}$ of the hairline. At the temporal region, the average density is $69.6 \mathrm{FU} / \mathrm{cm}^{2}$ and 97.6 hairs $/ \mathrm{cm}^{2}$. This translates to 1.4 hairs/FU.

\section{Age}

$$
\begin{aligned}
& 18-29(n=41) \\
& 30-39(n=35) \\
& 40-49(n=29) \\
& 50-59(n=33) \\
& 60-69(n=36) \\
& >70(n=35) \\
& \text { Average }(n=209)
\end{aligned}
$$

\begin{tabular}{|c|c|}
\hline Density & Hairs $/ \mathrm{cm}^{2}$ \\
\hline \multicolumn{2}{|c|}{$\begin{array}{l}\text { Frontal Hairline } \\
\qquad\left(\mathrm{FU} / \mathrm{cm}^{2}\right)\end{array}$} \\
\hline 85.2 & 120.0 \\
\hline 83.2 & 127.3 \\
\hline 79.3 & 118.2 \\
\hline 74.7 & 105.8 \\
\hline 76.8 & 103.0 \\
\hline 66.3 & 84.7 \\
\hline 77.8 & 110.0 \\
\hline
\end{tabular}

Hairs $/ \mathbf{c m}^{2}$ Temporal

Hairline

77.1

110.9

114.2

100.5

96.6

86.7

75.3

97.6

\section{Hairline Height}

The height of the hairline in non-AGA men does not change or recede significantly with age (see table below for the hairline height in Chinese men between the ages of 18 to 85), although the right temple tends to be slightly higher than the left.

$\begin{array}{lccc}\text { Age } & \begin{array}{c}\text { Frontal Hairline } \\ \text { Height } \\ (\mathbf{c m})\end{array} & \begin{array}{c}\text { Right Frontotemporal } \\ \text { Hairline Height } \\ (\mathbf{c m})\end{array} & \begin{array}{c}\text { Left Frontotemporal } \\ \text { Hairline Height } \\ (\mathbf{c m})\end{array} \\ 18-29(\mathrm{n}=41) & 6.7 & 8.8 & 8.8 \\ 30-39(\mathrm{n}=35) & 6.7 & 8.9 & 8.8 \\ 40-49(\mathrm{n}=29) & 7.2 & 9.2 & 9.2 \\ 50-59(\mathrm{n}=33) & 6.6 & 8.9 & 8.8 \\ 60-69(\mathrm{n}=36) & 6.7 & 8.9 & 8.8 \\ >70(\mathrm{n}=35) & 7.0 & 9.0 & 8.9 \\ \text { Average }(\mathrm{n}=209) & 6.8 & 8.94 & 8.87\end{array}$

\section{Hairline Shape}

In the study, we noted $55.5 \%$ of the hairlines were upsloping (Figure 5), 35.4\% were straight (Figure 6), and only $9.1 \%$ were downsloping (Figure 7).

\section{Face Shape}

The top three most common face shapes in Chinese based in Singapore were oval (46.9\%), round $(16.8 \%)$, and square $(13.9 \%)$.

\section{Hair Direction/ Widow's Peak/Lateral Mounds}

The hair direction flowed from the left to right in $59.8 \%$ of the cases, flowed right to left in $15.8 \%$, was radial in $10.5 \%$, and was anterior flowing in
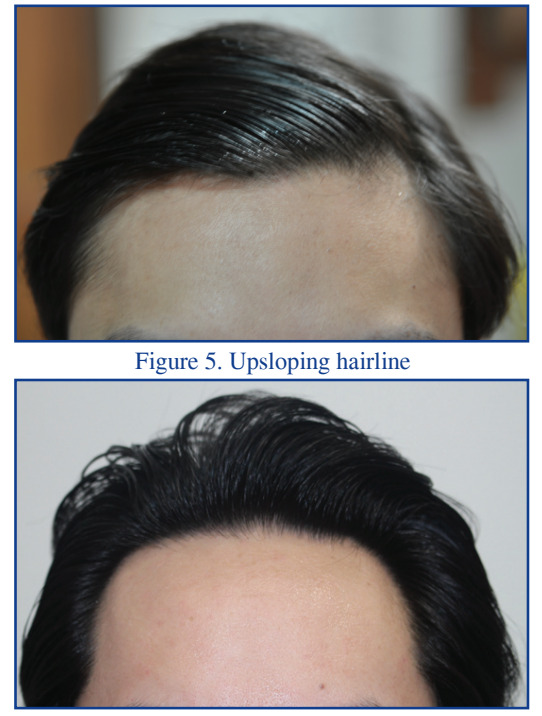

Figure 6. Straight hairline

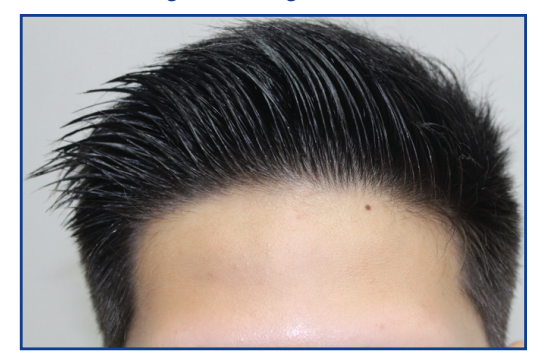

Figure 7. Downsloping (juvenile) hairline $13.9 \%$. The presence of a widow's peak was found in only $47.4 \%$ of men, and most Asian men did not have any lateral mounds (66\%). Cowlick at the frontal hairline was present in only $1 \%$ of the participants. 
Hairlines in Orientals from page 205

\section{Frontotemporal Angle and Hair Direction of the Temples}

The average frontotemporal angle is round in $62.2 \%$ and straight in $37.8 \%$. The average frontotemporal angle was slightly wider on the left at $97.7^{\circ}$ and $97.0^{\circ}$ on the right. The temples recede gradually with age but maintain a convex shape. Temple hair direction is shown in the table below:

$\begin{array}{lccc}\text { Temple Direction } & \text { Inferiorly } & \text { Posteriorly } & \text { Anteriorly } \\ \text { Right (\%) } & 22.0 & 71.3 & 6.7 \\ \text { Left (\%) } & 38.8 & 38.8 & 22.4\end{array}$

\section{Sideburns}

The average sideburn width was $1.43 \mathrm{~cm}$ and was at the level of the tragus in $45.5 \%$ on the left and $46.4 \%$ on the right. On the left, $29.7 \%$ were above the tragus with an average distance of $1.42 \mathrm{~cm}$, and $24.9 \%$ were below the tragus with an average distance of $2.33 \mathrm{~cm}$. On the right, $27.3 \%$ were above the tragus with an average distance of $1.39 \mathrm{~cm}$, and $26.3 \%$ were below the tragus with an average distance of $2.04 \mathrm{~cm}$. Knowledge of the norm is important in the re-creation of sideburns in hair transplant patients.

\section{Discussion}

The frontal hairline consists primarily of 1-hair grafts, which gradually convert to 2 -hair grafts as it moves $0.5-1 \mathrm{~cm}$ posteriorly.$^{16}$ Not surprisingly, the average density at the edge of the frontal hairline is 1.4 hairs $/ \mathrm{cm}^{2}$. It is important to create microirregularity in the frontal hairline for it to appear natural. ${ }^{16,17}$

In SE and E Asian men, lateral mounds are frequently absent and hence may not be important in hairline creation, although the creation of a widow's peak may appear to lower the hairline in younger patients. Shapiro (2013) recommends incisional densities of 25-35 follicular units per square centimeter (FU/ $\mathrm{cm}^{2}$ ) while Tsai et al. recommend a density of $30 \mathrm{FU} / \mathrm{cm}^{2}$ in Orientals. ${ }^{12,17}$ Other hair surgeons have been able to achieve 50$60 \mathrm{FU} / \mathrm{cm}^{2}$ with good results. ${ }^{13}$ It is important to avoid too much dense packing as this can result in poor growth.

As the patient ages, lesser hairline density is required for patient satisfaction as density declines progressively with age, even in non-AGA men. The average height of the MFP remains low at $6.8 \mathrm{~cm}$. It may be prudent to create a hairline higher than this so that the hairline continues to look natural as the patient ages.

Common shapes of the hairline seen in Orientals include slight upsloping of the hairline or straight. It is important to factor in the projected future hair loss based on family history and the patient's age, and to be extremely conservative in young patients by creating a higher, upsloping hairline $(7-9 \mathrm{~cm})$ as it consumes fewer grafts. ${ }^{13}$

If a lower hairline is desired, strong temples are needed to balance the look. The hair direction of the right temple tends to point posteriorly while the left temple typically points inferiorly or posteriorly, so it may be useful to follow Mother Nature, as vellus hairs are mostly still present. Generally, only $30 \mathrm{FU} / \mathrm{cm}^{2}$ is needed for the temples to look good due to the flat angle at which hairs exit the scalp here. Surgically created temples tend to be more angular compared to the rounded appearance of naturally occurring temples.
Face shape also plays an important role in hairline design. For example, a straight hairline will make a square face appear broader (Figure 8), a high hairline will make an oblong face appear even longer (Figure 9), and a low hairline makes a round face look shorter (Figure 10). A V-shape hairline may look good on Caucasians, but it does not look natural on East Asians. ${ }^{18}$
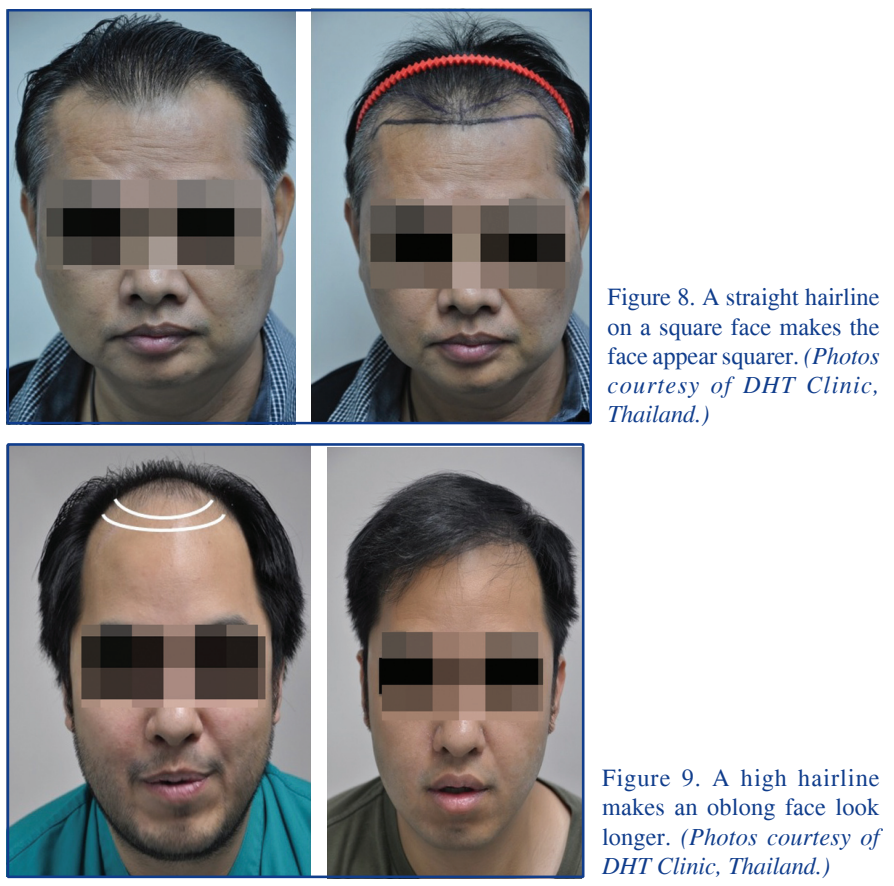

Figure 9. A high hairline makes an oblong face look longer. (Photos courtesy of

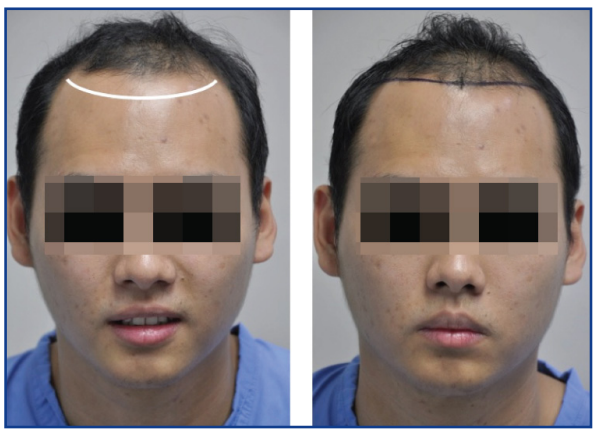
DHT Clinic, Thailand.)

There are various tools available that help to enhance the symmetry of the surgical hairline. Pathomvanich and $\mathrm{Ng}$ designed a laser light-leveling device that projects a curved laser beam over the planned hairline, which can just be outlined with an eyebrow pencil. ${ }^{19}$ Cole's "Aid to Hairline Design" measuring device also allows accurate measurement of the MFP and the frontotemporal angles. ${ }^{20}$

AGA can be inherited from both the maternal and paternal sides of the family. From the study, more and more patients are exposed to risk factors such as smoking and a westernized diet. ${ }^{6,21}$ Unsurprisingly, AGA is becoming a more common occurrence. Patients are also presenting at a younger age, and knowledge of the naturally occurring hairline will help both the surgeon and the patient to design and decide on a suitable hairline during hair restoration surgery. In addition, anticipated future hair loss and limitations of the donor reserves should be factored into the planning of a surgical hairline.

\section{Limitations of the Study}

Due to the fact that all the patients in the study had non-shaved hair, the patterns that we found may have varied from the litera- 
ture and we were unfortunately unable to assess the exit angle of the hair from the scalp due to the weight of the hair, which may be misleading.

\section{References}

1. Arca, E., et al. An open, randomized, comparative study of oral finasteride and 5\% topical minoxidil in male androgenetic alopecia. Dermatol. 2004; 209:117-125.

2. Khanna, M.. Hair transplant surgery. Indian J Plast Surg. 2008(Oct); 41(suppl):S56-S63.

3. Norwood, O.T. Male pattern baldness: classification and incidence. South Med J. 1975; 68:1359-1365.

4. Hamilton, J.B. Patterned loss of hair in man; types and incidence. Ann N Y Acad Sci. 1951; 53:708-728.

5. Takashima I., et al. Alopecia androgenica-its incidence in Japanese and associated conditions. In: C.E. Orfanos, W. Montagna, and G. Stutgen, eds. Hair Research Status and Future Aspects. Berlin: Springer-Verlag, 1981: 287-293.

6. Pathomvanich, D., et al. A random study of Asian male adrogenetic alopecia in Bangkok, Thailand. Dermatol Surg. 2002; 28:804-807.

7. Paik, J.H., et al. The prevalence and types of androgenetic alopecia in Korean men and women. Br J Dermatol. 2001; 145:95-99.

8. Su, L.H., and T.H. Chen. Association of adrogenetic alopecia with smoking and its prevalence among Asian men: a community-based survey. Arch Dermatol. 2007; 143:1401-1406.

9. Xu, F., et al. Prevalence and types of androgenetic alopecia in Shanghai, China: a community-based study. Br J Dermatol. 2009; 160:629-632.
10. Tang, P.H., et al. A community study of male androgenetic alopecia in Bishan, Singapore. Singapore Medical Journal. 2000; 41:202-205.

11. Hwang, S. Hair transplantation in East Asian Males. In: Hair Transplantation, 5th Edition. Unger and Shapiro, eds. Informa Healthcare, 2011; pp. 428-429.

12. Tsai, R., S. Lee, and H. Chan. The distribution of follicular units in the Chinese scalp: implications for reconstruction of natural-appearing hairline in Orientals. Dermatol Surg. 2002; 28:500-503.

13. Pathomvanich, D., and K. Imagawa, eds. Hair Restoration Surgery in Asians. Springer, 2010.

14. Beehner, M.L. Hairline design in hair replacement surgery. Facial Plast Surg. 2008; 24:389-403.

15. Unger, W. Recipient area hair direction and angle in hair transplanting. Dermatol Surg. 2004; 30:829-836.

16. Shapiro, R. Principles and techniques used to create a natural hairline in surgical hair restoration. Facial Plast Surg Clin N Am. 2004; 12:201-217.

17. Shapiro, R., and P. Shapiro. Hairline design and frontal hairline restoration. Facial Plast Surg Clin N Am. 2013; 351-362.

18. Pathomvanich, D. Hairline Design in Asians. Presented at the 5th Annual Conference of the Pan Asia Academy of FPRS. Bangkok, Thailand, 2015.

19. Pathomvanich, D., and B. Ng. Laser assisted hairline placement. Hair Transplant Forum Int'l. 2008; 18(5):169.

20. Cole, J. Aid to hairline design (AHD). Hair Transplant Forum Int'l. 2008; 18(5):173.

21. Lee, W., and H-J Lee. Characteristics of androgenetic alopecia in Asians. Ann Dermatol. 2012(Aug); 24(3):234-252.

\section{Wireless Follicular Dermatoscope}

\section{Wirelessly Capture Pictures:}

Twelve distinct levels of polarization. Observes and records in real time (30 FPS). Transmits within a range up to $20 \mathrm{ft}$. Built-in snapshot button. 126-FS-1

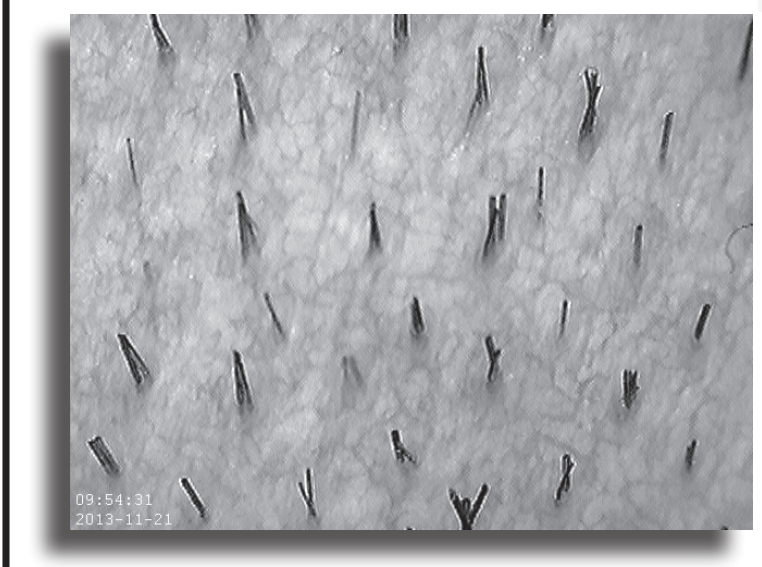

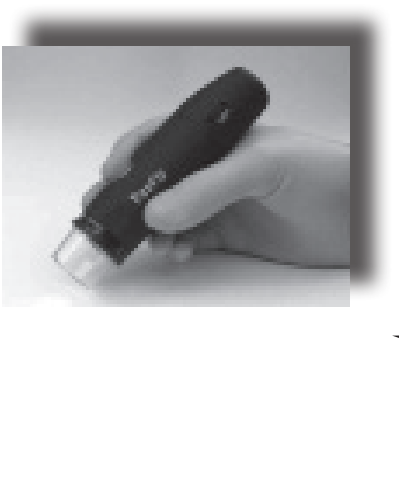

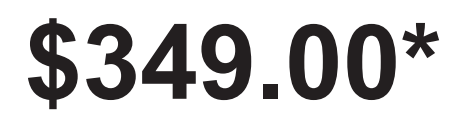

*When you mention this Forum ad.

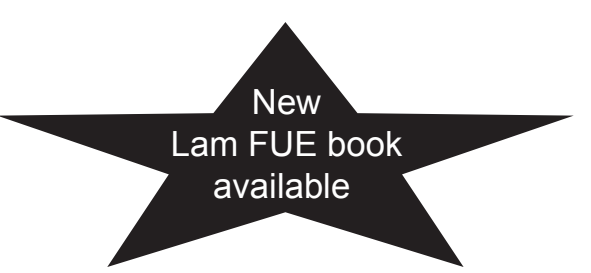

Kenny Moriarty

Vice President

Cell: 516.849 .3936

Kenny@georgetiemann.com

www.atozsurgical.com 